\title{
Simulación para formar en la competencia de valoración preanestésica: diseño común para residentes de primer año de anestesiología y enfermeras de anestesia
}

\author{
Manuel López-Baamonde, Alexis Martín-Calabuig, Miquel Coca, Marta Fargas-Alpuente, Lidia Gómez, \\ Raquel Bergé, Beatriz Tena, Carmen Gomar-Sancho
}

Introducción. La valoración preanestésica (VP) es una competencia compleja que debe adquirir el residente de anestesia tempranamente. La incorporación de enfermería especializada en la VP ha demostrado ser segura y eficaz. No existen recomendaciones para el entrenamiento en VP. La simulación puede ser una metodología apropiada para acortar el tiempo de adquisición de la competencia.

Objetivo. Describir detalladamente el diseño y aplicación de un taller de simulación para formar residentes de anestesiología y enfermeras posgraduadas en la competencia VP.

Sujetos y métodos. Taller de dos horas de duración con casos clínicos en formato video y ejecutados mediante técnica de role-playing. Los componentes fueron dos videos de grabación propia (el primero, de una VP con errores, y el otro, con una VP correctamente realizada), una plantilla para que cada alumno valorara en los videos los componentes técnicos y no técnicos de las competencias de la VP y una encuesta de satisfacción para alumnos e instructores. En el grupo de residentes se añadieron tres escenarios de VP de pacientes complejos.

Resultados. Se analizaron las encuestas de los 10 residentes de dos promociones y de 60 enfermeras. La satisfacción de los dos tipos de alumnos y de los instructores sobre el aprendizaje fue muy alta. En el caso de las enfermeras, más del $85 \%$ de las alumnas de las promociones que recibieron el taller superaron la estación VP de la evaluación clínica objetiva estructurada (ECOE) frente a sólo el 20\% de la promoción anterior.

Conclusiones. El taller de VP con formato video y role-playing permite entrenar la competencia VP a residentes de anestesia y enfermeras posgraduadas, mejorando el rendimiento de éstas en la ECOE.

Palabras clave. Competencias de enfermería. Educación. Residentes de anestesiología. Simulación. Valoración preanestésica. Videos formativos.

Simulation for training the competence pre-anesthetic assessment: a common design for first year anesthesia residents and anesthesia nurses

Introduction. Preoperative anesthetic assessment (PA) is a complex competence that anesthesia residents should acquire early in their training. Participation of specialized nurses in the PA is increasing since it is safe and efficacious. There is not an established curriculum in PA. Simulation may be an adequate methodology to train PA.

Aim. To describe in detail the design and application of a simulation workshop for training residents and postgraduate nurses in the competence PA.

Subjects and methods. Workshop of 2 hours duration, with clinical cases combining videotapes and role-playing. Workshop components were two homemade videos, one with a PA with errors and the other one with a correct PA, a checklist in which each participant assesses technical and non-technical components of the PA and a satisfaction survey for students and instructors. Three simulation scenarios of PA in complex patients were added for residents.

Results. 10 residents and 60 nurses participating in the workshop were surveyed. Satisfaction with the methodology applied was high for students and instructors. The percentage of passing the PA station of the final objective structured clinical examination (OSCE) of two promotions of nurses that had participated in the workshop was over $85 \%$ compared with only $20 \%$ of the previous promotion.

Conclusions. A simulation workshop based on videos and role-playing allows to train the competence PA in anesthesia residents and postgraduate nurses, improving the performance of the latter in the OSCE.

Key words. Anesthesiology residents. Education. Educational videotapes. Nurses' competences. Pre-anesthetic assessment. Simulation.

Servicio de Anestesiología y Reanimación y Área Quirúrgica. Hospital Clínic. Universitat de Barcelona. Barcelona, España.

Correspondencia:

Dr. Manuel López Baamonde. Servicio de Anestesiología. Hospital Clínic. Villarroel, 170. E-08036 Barcelona.

E-mail: lopez10@clinic.cat

Recibido: 13.12.17.

Aceptado: 19.12.17.

Conflicto de intereses: No declarado.

Competing interests: None declared.

(c) 2018 FEM 


\section{Introducción}

La valoración preanestésica (VP) es una entrevista médica orientada específicamente a valorar los riesgos de la cirugía y a preparar al paciente y los recursos necesarios antes de una intervención quirúrgica o exploración bajo anestesia. Hasta un $30 \%$ de los incidentes adversos declarados en el proceso quirúrgico muestran relación con una incorrecta o insuficiente VP [1] y, por tanto, debe formar parte de todo proceso quirúrgico [1,2]. La alta importancia de la VP para la seguridad de los pacientes quirúrgicos se pone de manifiesto por la obligatoriedad legal de llevarla a cabo (art. 10.6 de la Ley General de Sanidad de 21 de abril de 1986). El número de intervenciones quirúrgicas se incrementa progresivamente y los últimos informes señalan más de cinco millones de operaciones al año en España [3], de lo que se deduce la carga de trabajo y recursos que conlleva la VP.

Aunque la decisión sobre la anestesia, la preparación farmacológica y la obtención del consentimiento informado en la VP es responsabilidad de los médicos anestesiólogos, la incorporación de enfermeras especializadas en el equipo de anestesiología empieza a extenderse para aumentar la eficiencia. Existen evidencias de que este trabajo combinado de enfermería y anestesiólogos, en consultas simultáneas y contiguas, mantiene la seguridad del paciente y aumenta la calidad de la VP $[4,5]$.

La VP es una competencia difícil de adquirir porque requiere la integración de habilidades técnicas y no técnicas, con un importante peso de la comunicación con el paciente e interprofesional y de la toma de decisiones, todo ello encuadrado en una situación concreta del tipo de cirugía y del contexto en que se va a realizar. A menudo se requiere que los residentes de anestesiología realicen VP ya desde las primeras etapas formativas. Una revisión sobre 140 programas formativos de residentes de anestesiología encontró que la formación específica en VP es rara y, en cambio, los residentes asumen esta actividad muy tempranamente [6]. Creemos que esta situación persiste en la mayoría de programas de residentes.

Por otra parte, en España, la formación especializada de enfermería en anestesia no existe como tal y este rol de la enfermería depende de la organización de las instituciones. Existen formaciones universitarias de posgrado específicas de enfermería de anestesia, reanimación y tratamiento del dolor, básicamente en forma de másteres propios, con mayor o menor capacitación competencial. El Máster en Enfermería de Anestesia y Reanimación (MIAR) de la Universitat de Barcelona expone a sus alumnos a un contenido práctico capacitador, por lo que es necesario recurrir a una amplia red de centros. Al ser la incorporación de la enfermería a la VP limitada y desigual entre los centros, los responsables del máster fueron conscientes desde la primera evaluación clínica objetiva estructurada (ECOE) de este máster que las competencias para la VP no se obtenían. Así pues, nos encontramos con la necesidad de formar a los residentes de primer año y a las enfermeras del MIAR en la competencia VP.

Hasta la fecha no se ha descrito una metodología para enseñar la VP, que tradicionalmente se adquiere mediante la exposición repetida en la práctica real.

La simulación es una metodología formativa muy potente, complementaria del aprendizaje mediante práctica clínica, especialmente útil para la adquisición de habilidades no técnicas, y muy adecuada y utilizada en anestesiología [7] y en enfermería [8,9]. A pesar de su eficacia, la simulación tiene una extensión limitada por la carga de trabajo que conlleva su organización y realización [10]. Por ello, conviene diseñar fórmulas formativas en simulación, con componentes e instrumentos que puedan reproducirse ampliamente sin precisar cada vez el esfuerzo individual. Por otra parte, una competencia compartida entre distintas profesiones debería tener una formación similar.

El objetivo de este artículo es describir el diseño de un taller de simulación de VP para los residentes de anestesiología de primer año y las enfermeras posgraduadas, basado en la misma metodología que combina videos, role-playing y debriefing, y adaptado a ambas profesiones. Se describen también los resultados de la ECOE de las enfermeras antes y después de aplicar este taller en su programa de formación.

\section{Sujetos y métodos}

El diseño del entrenamiento por simulación de la VP siguió el proceso utilizado en toda enseñanza por simulación, con las fases [11] que se describen a continuación:

1. Establecimiento del grupo instructor: cuatro anestesiólogos instructores de simulación y dos residentes de tercer año que habían tenido entrenamiento repetido por simulación durante su formación, y dos enfermeros de anestesia profesores del MIAR con experiencia profesional en VP.

2. Establecimiento de los objetivos de la sesión para los dos tipos de participantes, residentes y enfermeras, con aspectos comunes y diferenciales. 
Los comunes eran la valoración de forma exhaustiva, sistemática y orientada de todos los ítems que conforman la VP (información al paciente y aclaración de sus dudas, preparación farmacológica y no farmacológica, información sobre el dolor postoperatorio, documentación clínica de la VP, previsión de las necesidades dependientes del equipo quirúrgico y aptitudes comunicativas en la relación profesional-paciente). Los aspectos específicos para los residentes eran el establecimiento del plan anestésico y obtención del consentimiento informado, y para las enfermeras, medidas de higiene, hábitos de salud y preparación para la anestesia.

3. Características de la población diana de la formación: los cinco residentes de primer año de anestesiología de dos promociones (cinco por año) que todavía no habían tenido ninguna sesión de simulación en el servicio y 60 enfermeras matriculadas en el MIAR. Todos habían recibido las bases teóricas de la VP.

4. Elaboración de dos videos caseros de VP simulada para residentes y dos para enfermeras, con igual guión, excepto para aquellos componentes específicos de la competencia para cada profesión. En estos videos, un actor simulado hace de paciente, y otro, de anestesiólogo o enfermera, respectivamente.

En el primer video para ambas profesiones, la VP se realiza de forma incompleta, dirigida a la recogida de datos y no a su análisis, realizando un registro incompleto y no dirigido de los antecedentes patológicos médicos y quirúrgicos, alergias, antecedentes transfusionales, con deficiencias en la valoración del estado funcional, en la comunicación con el paciente y en la información que se le proporcionaba. En el caso de las enfermeras, el video de VP errónea contenía también la asunción de competencias propias del médico y, en el caso del video para residentes, se observaban defectos en la obtención del consentimiento informado y la asunción de competencias informativas del cirujano.

El segundo vídeo muestra una VP realizada de forma sistemática, exhaustiva, orientada y analítica, con integración de toda la información para realizar una valoración global del paciente en su estado funcional y las posibles repercusiones en la anestesia y cirugía, así como una actitud y comunicación asertiva, informativa y tranquilizadora hacia el paciente, evitando actitudes paternalistas y la intromisión en competencias no propias. Los actores de los videos o enfermeras son miembros del equipo instructor con los que los resi- dentes y enfermeras todavía no han tenido ningún contacto previo.

5. Diseño de una plantilla con cada una de las competencias y aptitudes de la VP, con formato de lista de comprobación o checklist (Tabla I). Durante la visualización de los videos, los residentes y enfermeras deben valorar individualmente la ejecución de cada componente de la competencia. La plantilla les indica observar activamente los distintos componentes de la competencia. En el caso de las enfermeras, esta misma lista de comprobación se utilizaba para la valoración en la estación de la ECOE.

6. Distribución y cronología del taller: los residentes, el grupo de cinco, permanecen juntos durante todo el taller. Los 60 alumnos de enfermería se distribuyen en dos grupos de 30 en aulas diferentes para mejorar el control y la comunicación durante la discusión tras la visualización de los videos. A su vez, se organizan en subgrupos de 6-8 alumnos para discutir y analizar los videos y participar en el debriefing de forma colaborativa.

Se estableció una duración de dos horas que se distribuyeron en las siguientes fases (para residentes y enfermeras):

- 10 minutos. Briefing o explicación del taller y su misión formativa y organización, recuerdo de los objetivos de la VP, entrega de la plantilla con las competencias detalladas por componentes técnicos y no técnicos que deben valorar mientras visualizan los videos.

- 10 minutos. Visualización del primer video de una VP con defectos. Durante su visionado, los participantes deben analizar y apuntar en la plantilla los errores o defectos que observan.

- 10 minutos. Discusión bajo la dirección de los instructores para analizar los fallos objetivados en el video. En el caso de las enfermeras, esta discusión se realiza dentro de cada subgrupo de 6-8 alumnos para participar en el debriefing de forma colaborativa.

A partir de este momento, el taller para enfermeras y residentes se desarrolla de forma diferente. En el caso de las enfermeras:

- 45 minutos. Debriefing o discusión abierta y reflexiva en el que los distintos grupos de 6-8 alumnos analizan en conjunto los defectos o fallos por apartados, moderados por el instructor para seguir el orden cronológico de la tabla I, ajustándose al orden cronológico que sigue el video. 
Tabla I. Lista de comprobación a cumplimentar durante la visualización de los videos de valoración preanestésica. Los componentes específicos de enfermería se señalan con una letra ' $E$ ', y los de anestesiología, con una letra ' $A$ '. La valoración se realizaba sobre la solicitud del servicio de cirugía siguiente: 'Paciente Fernando L.S. Historia clínica n. 101100. 55 años. Alergia a tramadol y frutos secos. Fumador. Antecedentes: hipertensión arterial, dislipidémico, hernia de hiato. Intervenciones quirúrgicas previas: politraumatismo con necesidad de fijación cervical por fractura vertebral'.

Saluda y se presenta

Confirma nombre y datos del paciente y operación propuesta

Indaga alergias, sospecha de alergia al látex

Indaga sobre hábitos tóxicos y sus consecuencias

Indaga sobre anestesias y antecedentes quirúrgicos previos

Indaga sobre secuelas de la cirugía cervical

Indaga sobre antecedente transfusionales

Calcula el índice de masa corporal o al menos determina si existe obesidad

Indaga sobre medicación de ácido acetilsalicílico, bisoprolol y su control, y otros tratamientos farmacológicos

Indaga sobre medicamentos de parafarmacia (homeopatía, herbolarios)

Indaga sobre el control y consecuencias de la hipertensión arterial, especialmente cardíacas y renales

Indaga sobre el dolor retroesternal y la hernia de hiato

Indaga sobre síntomas respiratorios y hábito tabáquico

Indaga sobre síntomas de alteraciones de la coagulación y riesgo trombótico

Indaga sobre estado funcional respecto a la actividad física

Establece riesgo según la escala ASA
Indaga sobre la ansiedad y tranquiliza al paciente adecuadamente

Valoración de la vía aérea (escala de Mallampati, distancia tiromentoniana, movilidad cervical); estado dental y protésis

E. Comprueba que se han realizado las pruebas complementarias protocolizadas

A. Valora las pruebas complementarias

A. Prescribe profilaxis antiemética, antitrombótica y ansiolisis

A. Prescribe adaptación de los tratamientos farmacológicos

E. Da explicaciones de cómo será el procedimiento

A. Informa sobre el dolor postoperatorio esperado y su tratamiento

E. Instruye al paciente sobre técnicas de alivio del dolor postoperatorio

E. Informa al paciente sobre medidas de higiene y preparación para la cirugía

Resolución de dudas que plantea el paciente y confirmación de que ha comprendido la información

A. Explicación del circuito anestésico-quirúrgico

Incide en educación sanitaria y hábitos de vida saludables

E. Indica que la visita continuará con el anestesiólogo, que le informará del procedimiento

A. Obtiene el consentimiento firmado y da indicaciones de cómo continuará la valoración preanestésica

Se despide correctamente

La plantilla consta de tres columnas: lo realiza (sí/no); correcta o incorrectamente; comentarios para el debriefing con el alumno/a.

- 10 minutos. Visualización del video de la VP realizada correctamente para analizar y apuntar en la plantilla los aspectos que consideran relevantes.

- 20 minutos. Discusión general y extracción de conclusiones activamente por parte de los alumnos. En esta fase se comparan ambos videos y se llega a la resolución de los problemas o defectos planteados.

En el caso de los residentes:

- 10 minutos. Visualización del segundo video de VP correcta.

- 15 minutos. Debriefing y extracción de conclusiones a partir de la comparación de los dos videos.
- 30 minutos. Tres escenarios de simulación roleplaying de situaciones conflictivas frecuentes en la VP: paciente muy ansioso, paciente que insiste en obtener la información quirúrgica y paciente pluripatológico desconfiado que rechaza la técnica anestésica propuesta. El diseño de estos tres escenarios seguía el esquema clásico de los escenarios de simulación: el paciente era un actor simulado y el atrezzo o decorado y la duración de la VP eran los habituales en nuestro centro. Todos los residentes observaban la ejecución de los casos simulados de sus compañeros que eran filmados.

- 45 minutos. Debriefing apoyado por secuencias de la filmación de las simulaciones. Encuesta de 
satisfacción y valoración de la sesión por parte de los alumnos y todo el equipo de simulación.

En ambos grupos, el taller finalizaba con cinco minutos para cumplimentar la encuesta de satisfacción a los alumnos y de los instructores (Tabla II).

Se evaluaron los resultados obtenidos en la evaluación final por los alumnos del máster de enfermería de los cursos 2014-2015 y 2015-2016 que habían recibido el taller y se compararon con los del curso 2013-2014 que no lo habían recibido.

Se analizaron las encuestas de satisfacción al final del taller de las enfermeras del curso 2014-2015 y de los residentes.

\section{Resultados}

Este taller se ha aplicado a 10 residentes de anestesiología de primer año de dos promociones (20142015 y 2015-2016) y a tres promociones de alumnas del MIAR (2014-2015, 2015-2016 y 2016-2017).

Los residentes tenían una edad media de 26,3 \pm 2,2 años. La encuesta de satisfacción de los 10 residentes alcanzó una satisfacción global de 4,6 puntos sobre 5. La comparación de los dos videos -correcto e incorrecto- mediante la plantilla y los casos simulados fueron considerados muy útiles en opinión de todos. El debriefing de los videos y el de los casos simulados se consideraron igualmente muy útiles. Opinaron que les hubiera gustado simular más casos y valoraron muy favorablemente la participación de residentes de tercer año como instructores.

Los instructores de los residentes puntuaron la valoración global del taller con un 4,3. Como comentario libre, añadieron que el tiempo programado para el debriefing de los casos simulados no les permitió utilizar las grabaciones en la extensión que deseaban. Consideraron que los residentes de tercer año estaban capacitados para impartir este taller.

La encuesta de satisfacción de las enfermeras del MIAR fue cumplimentada por el $91 \%$ de alumnas ( 55 de 60 ). Su edad media era de 36,4 $\pm 8,6$ años. El $98 \%$ valoraban como muy útil la utilización de casos simulados en formato video en el aprendizaje de situaciones clínicas reales, además de obtener un mejor razonamiento clínico, pensamiento crítico y aprendizaje grupal.

El 96\% afirmaban que es útil comparar ambas simulaciones, la que contiene errores y posteriormente la correctamente realizada. El 100\% afirmaban que la realización del debriefing grupal tras la visualización de los casos simulados promueve un aprendizaje colectivo más efectivo.
Tabla II. Encuesta de satisfacción de los participantes en el taller.

Edad (años):

$¿$ Has realizado anteriormente clases o cursos con casos simulados en formato video?

Sí / No

¿Cómo valorarías la utilización de casos simulados en videos para el aprendizaje de situaciones clínicas reales?

De 0 (inútil) a 5 (muy útil)

¿Crees que se consigue razonamiento clínico, pensamiento crítico y aprendizaje con la realización de casos simulados en video?

Sí / No / No sé

¿Crees que es útil el empleo de un caso simulado erróneo para identificar los fallos y resolver los posibles déficits de éste?

Sí / No / No sé

¿Crees que la comparación de una actuación incorrecta con una correcta es útil en la planificación y resolución de los objetivos?

Sí / No / No sé

¿Crees que el debriefing (análisis y discusión) grupal tras la visualización de los videos promueve el aprendizaje?

Sí / No / No sé

¿Qué importancia le das al uso de casos clínicos simulados de valoración preanestésica como método de aprendizaje individual y colectivo para abordar situaciones clínicas reales?

De 0 (ninguna importancia) a 5 (máxima importancia)

¿En qué proporción del aprendizaje que has obtenido ha influido el debriefing?

De $0 \%$ a $100 \%$

Sugiere iniciativas en un taller de casos simulados para mejorar el aprendizaje o comprensión de las situaciones clínicas reales de la valoración preanestésica:

Preguntas específicas en la encuesta de residentes:

¿Cómo valoras la participación de residentes de tercer año como instructores?

De 0 (inútil) a 5 (muy útil)

¿Cómo valoras los casos simulados de pacientes complejos para aproximarte a la situación clínica real?

De 0 (inadecuados) a 5 (muy adecuados)

Preguntas específicas para los instructores:

¿Cómo valoras la participación de residentes de tercer año como instructores?

De 0 (inútil) a 5 (muy útil)

Comenta aspectos del diseño y desarrollo del taller que creas mejorables:

Respecto a los resultados en la evaluación final del MIAR comparamos las calificaciones de la promoción 2013-2014, que fue previa a la instauración del taller presentado, con las dos promociones siguientes, que sí lo tuvieron. En la evaluación de la promoción 2013-2014, sólo el 20\% de los alumnos pasaron la estación VP de la ECOE final, lo que contrastaba con la alta tasa de un 95\% de aprobados en las preguntas sobre VP del examen teórico MCQ.

En las dos promociones siguientes, 2014-2015 y 2015-2016, que recibieron el taller, la tasa de aprobados en el examen teórico permaneció igualmente alta (superior al 96\% y 97\%, respectivamente), pero 
las tasas de aprobados en la estación VP de la ECOE fueron superiores al 90\% en el curso 2014-2015 y superior al 85\% en el curso 2015-2016.

El taller de VP así diseñado se ha integrado tanto en el programa de formación por simulación de los residentes de anestesiología de nuestro centro como al MIAR.

\section{Discusión}

La VP es un aspecto clave para la seguridad y resultados de los procedimientos quirúrgicos [1-3] y para la eficiencia organizativa de la cirugía [12-14]. La VP es una competencia compleja que integra el conocimiento de los cambios fisiopatológicos específicos de la anestesia y cirugía, valoración y juicio clínicos, capacidad de interpretar pruebas complementarias, valoración del riesgo anestésico-quirúrgico y el proceso de integración de toda la información para determinar el estado funcional del paciente en relación a un proceso quirúrgico específico y la elaboración de un plan anestésico. La VP, además, requiere competencia en habilidades de comunicación con el paciente e interprofesional. La VP disminuye la ansiedad perioperatoria y permite la obtención de un consentimiento informado de calidad.

La realización de la VP en todo paciente quirúrgico es obligada por ley en España y en la mayor parte de Europa y supone una actividad muy prevalente, ya que el número de intervenciones quirúrgicas anuales en España supera los cinco millones [5].

La incorporación de enfermería especializada a las consultas de preanestesia como parte del equipo de anestesiología se considera actualmente una forma de aumentar la eficiencia de esta actividad y además se ha demostrado que incrementa su calidad [4]. Sin embargo, es un nuevo rol de enfermería que todavía se está aplicando de forma escasa e irregular en los diferentes centros sanitarios. La transición de actividades clásicamente médicas a personal de enfermería precisa su especialización, que en España sólo está organizada para algunas especialidades. El MIAR de la Universitat de Barcelona incluye la competencia de VP de enfermería en su programa. Las prácticas clínicas se realizan en diversos hospitales asociados y se valora su consecución mediante una ECOE final. La dirección del MIAR detectó que el porcentaje de superación de la estación VP del 20\% era insostenible y difícil de mejorar durante las rotaciones clínicas por la escasez de centros donde la enfermería se ha incorporado a esta actividad. Sin embargo, constituye un rol nuevo que la enfermería de anestesia debe asumir y, por tanto, que debemos enseñar. De este problema surgió la necesidad de un método de enseñanza que se aproximase al máximo a una VP real, y un taller de simulación con role-playing y videos se consideró lo más factible para un grupo numeroso de 60 enfermeras matriculadas en cada promoción.

Por otra parte, la compleja competencia de la VP la asume con mucha frecuencia el residente desde sus primeras fases $[2,6]$, y aunque se desconoce si esto afecta la calidad [15], todos los programas de formación de residentes la incluyen, aunque no abordan cómo entrenarla y clásicamente se adquiere con los años de experiencia clínica.

En la revisión de la bibliografía sólo hemos encontrado dos artículos que tratan aspectos de la metodología para entrenar en VP. Uno de ellos fue publicado por nuestro grupo comparando la clase convencional con la metodología de discusión de casos-problema para la enseñanza de la VP a residentes de primer año [16]. El otro se limita a sugerir, sin aplicarla, que la simulación podría ser una buena técnica para entrenar a personal no médico, enfermeras o técnicos en VP [17]. Por ello hemos considerado que podría ser útil una descripción detallada del diseño y ejecución del taller realizado por nuestro equipo, de forma que facilitara el diseño de este entrenamiento a otros grupos docentes.

Un aspecto que nos pareció importante es que si la VP es una competencia que deben compartir multidisciplinariamente anestesiólogos y enfermeras de anestesia, deberíamos diseñar un método de formación integrado y coherente para ambos grupos.

El diseño del taller que presentamos se fundamentó en metodología docente ampliamente aceptada. La simulación es la metodología docente más potente para aproximarse a la situación real y acortar el tiempo de aprendizaje, en especial para habilidades no técnicas. Se adecua a la actividad asistencial y es complementaria a las posibles carencias de exposición clínica real $[18,19]$. El role-playing y los 'pacientes simulados' son ampliamente utilizados y reconocidos para la entrevista clínica en general [20], que tiene un cometido superponible a la VP y, por ello, decidimos aplicar esta técnica para la enseñanza de la VP. La utilización de videos para la formación en ciencias de la salud, y específicamente para competencias relacionadas con la entrevista clínica, se considera útil $[21,22]$. La tecnología actual permite elaborar videos fácilmente y con bajo coste para un equipo docente. La plantilla que los participantes debían cumplimentar durante la visualización de los videos estaba diseñada como checklist o lista de comprobación para el análisis de los distintos componentes de la VP, preguntándoles 
sobre los aspectos relevantes o más complejos de la actuación correcta o incorrecta en la VP, y sirvió de guía del debriefing de los videos. Las listas de comprobación se han mostrado útiles para la enseñanza por simulación y videos [23,24] y consideramos que, en este taller, la lista fue clave para la utilidad de los videos. Esta plantilla no sólo permitía preparar los puntos del debriefing, sino que tenía un papel formativo adicional al organizarse por los componentes clave de la VP y en un orden lógico y coherente. Esta misma lista de comprobación es la que se utiliza para la valoración de la estación VP en la ECOE del MIAR.

El debriefing, la parte fundamental de la simulación que cambia realmente al participante, fue extenso y estructurado, tanto después de la visualización de los videos como tras la simulación en los tres escenarios de VP en el caso de los residentes. Residentes y enfermeras participantes en el taller evaluaron muy bien los videos como herramienta docente y especialmente la comparación de las actuaciones erróneas y correctas. Para grupos numerosos, como fue el caso de las alumnas del máster, en donde no es posible hacer casos simulados, la visualización activa de videos permite un debriefing extenso y concluyente sobre la actuación profesional en la competencia VP. Las ventajas del debriefing colaborativo [25] permitió aplicar esta técnica a los grupos numerosos de enfermería.

La combinación descrita de recursos docentes ampliamente aceptados permitió hacer un taller que creemos que está al alcance de cualquier equipo docente.

La limitación de nuestra propuesta es la imposibilidad de demostrar objetivamente que este taller haya sido eficaz para mejorar la competencia clínica de los residentes y enfermeras en la VP real. Esta evaluación precisaría la observación de la realización de la VP en distintos pacientes, lo que no hemos hecho. Sólo podemos afirmar que este taller fue factible y satisfactorio para todos los participantes y que, en el caso de las enfermeras, mejoró ostensiblemente el rendimiento en la ECOE. La falta de valoración de los resultados de la formación sobre la práctica clínica real ocurre en todos los métodos docentes utilizados en ciencias de la salud. Quizá el siguiente paso para valorar la influencia de nuestro taller de VP sea aplicar mini-CEX (Mini-Clinical Evaluation Exercise), que se basa en observar varias ejecuciones reales por distintos evaluadores que posteriormente da un feedback positivo [20,26-28].

Los tres escenarios de VP precisaban que los residentes lograran los objetivos de la VP en situaciones complejas, pero muy frecuentes en la práctica real, y el debriefing posterior permitió ahondar en la comunicación, obtención y transmisión de información a pacientes complejos. La observación de la filmación de los escenarios permitió analizar habilidades complejas y las actitudes. La participación activa de los residentes de tercer año como instructores supuso un beneficio formativo adicional para ellos, que se preparan para utilizar metodologías docentes innovadoras en la formación de residentes, y fue muy bien valorada.

Los profesionales reconocen bien sus necesidades formativas y por ello su opinión favorable sobre una actividad formativa tiene valor. La encuesta realizada al final del curso no sólo pretendió valorar la satisfacción, sino la percepción del alumno respecto a las distintas partes del taller. Por ello indagamos sobre la utilidad de los videos, tanto en su aproximación a la realidad como en la modalidad de comparar actuaciones incorrectas y correctas en la misma situación. Para más del 96\%, los videos fueron útiles para estos objetivos y el 100\% de las enfermeras consideró el debriefing tras la visualización de los videos como la parte más potente para el aprendizaje colectivo efectivo, lo que refuerza la importancia universalmente reconocida del debriefing. Destacamos que la satisfacción fue igualmente alta para residentes y enfermeras, que presentaban una diferencia de edad de más de 10 años de media.

La opinión del equipo docente fue muy buena y con el taller ya diseñado la carga de trabajo se considera muy apropiada. Ha quedado establecido de manera permanente en el máster y en el programa de formación de residentes.

Este artículo es la primera descripción de una formación estructurada de la compleja competencia de VP para residentes y para enfermeras especializadas; integra distintos componentes de la simulación, que ha sido fácilmente aplicable en nuestro medio y creemos que puede ser extendida de forma amplia. Además, el esfuerzo del diseño es más eficiente porque engloba dos profesiones y aumenta la coherencia en la formación de un equipo interprofesional.

\section{Bibliografía}

1. Schonberger RB, Rosenbaum SH, eds. Medically complex patients. Anesthesiol Clin 2016; 34: 633-820.

2. Yen C, Tsai M, Macario A. Preoperative evaluation clinics. Curr Opin Anaesthesiol 2010; 23: 167-72.

3. Coduras-Martínez A, Del Llano-Señarís JE. La sanidad española en cifras 2016. Madrid: Círculo de la Sanidad; 2017. URL: http://www.cesm.org/wp-content/uploads/2017/03/ LIBRO_SANIDAD_ESPANOLA_EN_CIFRAS_2016.pdf.

4. Mata J, Moral V, Moya M, Nolla M. ¿Es eficaz, seguro y eficiente una consulta preanestésica en equipo con enfermería? Rev Esp Anestesiol Reanim 2007; 54: 279-87. 
5. Nicholson A, Coldwell CH, Lewis SR, Smith AF. Nurse-led versus doctor-led preoperative assessment for elective surgical patients requiring regional or general anaesthesia. Cochrane Database Syst Rev 2013; 12: CD010160.

6. Tsen LC, Segal S, Pothier M, Bader AM. Survey of residency training in preoperative evaluation. Anesthesiology 2000; 93 : 1134-7.

7. Maestre JM, Sistac-Ballarín JM, González A, Luis JC, SáezFernández A. Grupo de Trabajo en Simulación Clínica de la Sección de Docencia y Formación de la SEDAR. La simulación clínica y nuestra nueva realidad. La necesidad de aunar esfuerzos. Rev Esp Anestesiol Reanim 2012; 59: 233-4.

8. Fernández-Ayuso D, Del Campo-Cazallas C, FernándezAyuso RM. Aprendizaje en entornos de simulación de alta fidelidad: evaluación del estrés en estudiantes de enfermería. Educ Med 2016; 17: 25-8.

9. MacLean S, Kelly M, Geddes F, Della P. Use of simulated patients to develop communication skills in nursing education: an integrative review. Nurse Educ Today 2016; 48: 90.

10. Gomar-Sancho C, Palés-Argullós J. ¿Por qué la simulación en la docencia de las ciencias de salud sigue estando infrautilizada? Educ Med 2011; 14: 101-3.

11. INACSL Standards Committee. INACSL standards of best practice: Simulation. Simulation design. Clinical Simulation in Nursing 2016; 12: S5-12.

12 Practice Advisory for Preanesthesia Evaluation. An updated report by the American Society of Anesthesiologist Task Force on Preanesthesia evaluation. Anesthesiology 2012; 116: 522-38.

13. García-Miguela FJ, Peyró R, Mirón-Rodríguez MF. Valoración anestésica preoperatoria y preparación del paciente quirúrgico Rev Esp Anestesiol Reanim 2013; 60 (Supl 1): 11-26.

14. Correll DI, Bader AM, Hull MW, Hsu C, Lawrence C, Tsen LC, et al. Value of preoperative clinic visits in identifying issues with potential impact on operating room efficiency. Anesthesiology 2006; 105: 1254-9.

15. Kim D, Lee, SN, Kim DC, Lee J, Ko S, Lee SK, et al. The preanesthetic interview by anesthesiology residents: analysis of time and content. Korean J Anesthesiol 2012; 62: 220-4.

16. Carrero E, Gomar C, Penzo W, Rull M. Comparison between lecture-based approach and case/problem-based learning discussion for teaching pre-anesthetic assessment. Eur J Anaesthesiol 2007; 24: 1008-15.
17. Van Klei WA, Hoff R. The simulated setting for testing competencies; task shifting in outpatient anaesthesia. Ned Tijdschr Geneeskd 2013; 157: A5905.

18. Gomar-Sancho C, Palés-Argullós J. Características de la educación médica basada en simulaciones. In Millán J, Palés J, Morán J, eds. Educación médica. Madrid: Editorial Médica Panamericana. 2015; p. 263-71.

19. Lorello GR, Cook DA, Johnson RL, Brydges R. Simulationbased training in anaesthesiology: a systematic review and meta-analysis. Br J Anaesth 2014; 112: 231-45.

20. Alyami H, Su'a B, Sundram F, Alyami M, Lyndon MP, Yu TC, et al. Teaching medical students history taking content: a systematic review. Am J Educ Res 2016; 4: 227-33.

21. Delás J, Penzo W, Delás A, González-Cardona R, Morcillo C, Martín G. Ver, oír y aprender. Una filmación educativa para la enseñanza de habilidades en exploración física. FEM 2014; 17: 93-7.

22. Lee WS, Hwang JY, Lim JE, Suh SY, Park KH, Sung NJ. The effect of videotaping students' interviews with patients for interview skill education. Korean J Fam Med 2013; 34: 90-7.

23. Luna-Villanueva E, De los Santos-Rodríguez M, Sierra-Basto G, González-Arriaga CR, Zamora-Graniel FG. Retroalimentación integral (debriefing) oral y asistida por video en simulación de reanimación cardiopulmonar avanzada: estudio piloto. FEM 2015; 18: 139-47.

24. Bernard AW, Ceccolini G, Feinn R, Rockfeld J, Rosenberg I, Thomas L, et al. Medical students review of formative OSCE scores, checklists, and videos improves with student-faculty debriefing meetings. Med Educ Online 2017; 22: 1324718

25. Guinez-Molinos S, Martínez-Molina A, Gomar-Sancho C Arias-González VB, Szyld D, García-Garrido E, et al. A collaborative clinical simulation model for the development of competencies by medical students. Med Teach 2017; 3: 195-202.

26. Weller JM, Jolly B, Misur MP, Merry AF, Jones A, Crossley JGM, et al. Mini-clinical evaluation exercise in anaesthesia training. Br J Anaesth 2009; 102: 633-41.

27. Baños JE, Gomar-Sancho C, Grau-Junyent LM, Palés-Argullós J, Sentí M. El mini-CEX como instrumento de evaluación de la competencia clínica. Estudio piloto en estudiantes de medicina. FEM 2015; 18: 155-60.

28. Baños JE, Gomar-Sancho C, Guardiola E, Palés-Argullós J. La utilización del Mini Clinical Evaluation Exercise (mini-CEX) en estudiantes de medicina. FEM 2015; 18: 417-26. 